\title{
Paternal age at birth and high-functioning autistic-spectrum disorder in offspring
}

Kenji J. Tsuchiya, Kaori Matsumoto, Taishi Miyachi, Masatsugu Tsujii, Kazuhiko Nakamura,

Shu Takagai, Masayoshi Kawai, Atsuko Yagi, Kimie Iwaki, Shiro Suda, Genichi Sugihara, Yasuhide Iwata, Hideo Matsuzaki, Yoshimoto Sekine, Katsuaki Suzuki, Toshirou Sugiyama, Norio Mori and Nori Takei

\section{Background}

Previous studies have reported the association between advanced paternal age at birth and the risk of autisticspectrum disorder in offspring, including offspring with intellectual disability.
Aims
To test whether an association between advanced paternal age at birth is found in offspring with high-functioning autistic-spectrum disorder (i.e. offspring without intellectual disability).

\section{Method}
A case-control study was conducted in Japan. The participants consisted of individuals with full-scale $I Q \geqslant 70$, with a DSM-IV autistic disorder or related diagnosis. Unrelated healthy volunteers were recruited as controls. Parental ages were divided into tertiles (i.e. three age classes). Odds ratios and 95\% confidence intervals were

estimated using logistic regression analyses, with an adjustment for age, gender and birth order.

\section{Results}

Eighty-four individuals with autistic-spectrum disorder but without intellectual disability and 208 healthy controls were enrolled. Increased paternal, but not maternal, age was associated with an elevated risk of high-functioning autisticspectrum disorder. A one-level advance in paternal age class corresponded to a 1.8-fold increase in risk, after adjustment for covariates.

\section{Conclusions}

Advanced paternal age is associated with an increased risk for high-functioning autistic-spectrum disorder.

\section{Declaration of interest}

None. Funding detailed in Acknowledgements.
Recent large-scale studies have reported that advanced paternal age at birth is a risk factor for autistic-spectrum disorder in their offspring. ${ }^{1-3}$ This finding has been interpreted as indicative of an increased likelihood of mutagenesis in the paternal germ line due to ageing. ${ }^{4}$ However, a more recent study failed to confirm the association. ${ }^{5}$ Furthermore, this association remains inconclusive, since it has only been reported thus far in studies mainly from Western countries (e.g. Denmark and the USA). Inconsistent findings may also stem from methodological differences. Studies have suggested that some characteristics may be associated with the risk of autistic-spectrum disorder, and that if these factors are not adequately allowed for, erroneous conclusions may be reached. These include birth order and socio-economic status. Autism has been associated independently with the highest birth order, ${ }^{6,7}$ and higher social class associated with an increased likelihood of having a diagnosis of autism. ${ }^{8}$ However, recent large-scale studies did not adjust for these factors. ${ }^{2,3}$

Furthermore, control for IQ or exclusion of those individuals with autistic-spectrum disorder who have intellectual disability has not been considered in previous studies. Advanced paternal age at birth has been demonstrated to be associated with an increased occurrence of offspring with developmental disabilities, as reflected in special education placement, ${ }^{9}$ and neuropsychiatric conditions such as epilepsy ${ }^{10}$ and schizophrenia. ${ }^{11,12}$ Moreover, intellectual ability has been shown to have an inverted U-shape relationship to paternal age at birth; ${ }^{13,14}$ that is, offspring of younger as well as older fathers tend to have lower IQ scores. On the other hand, $40-62 \%$ of people with autism have been reported to have intellectual impairment. ${ }^{15}$ Together, these findings point to the possibility that the association found in previous studies of samples with autistic-spectrum disorder, irrespective of IQ, might be attributable to linkage of advanced paternal age with the risk of cognitive impairment rather than with the risk of autistic-spectrum disorder per se.

In our study, we therefore investigated whether the association between advanced paternal age at birth and the risk of autisticspectrum disorder reported would be reproducible in a Japanese sample of individuals with high-functioning autistic-spectrum disorder but without intellectual impairment.

\section{Methods}

This study was conducted as a series of projects launched to investigate pregnancy- and birth-related events as risk factors for mental illnesses in Japan. The projects were designed to exclusively utilise the Mother and Child Health Handbook, a set of notes covering a wide range of information, including pregnancy and delivery events, newborn characteristics, as well as parental data including the dates of birth of the parents.

\section{Participants}

We sought the participation of individuals with a diagnosis of autistic-spectrum disorder but without intellectual disability who were admitted to, or referred to, the University Hospital of the Hamamatsu University School of Medicine during the period 1 October 2004 to 31 March 2006, or who were, during the same period, registered as members of a self-help organisation, 'AspeErude no Kai' (the Association for Asperger Syndrome, Autism, and Learning Disorders; Nagoya, Japan). To select individuals without intellectual disability, IQ was evaluated for those whose parents consented to participation in this study, and who themselves also consented to participate. 
The Japanese version of the Wechsler Adult Intelligent Scale Revised (WAIS-R) ${ }^{16}$ or the Wechsler Intelligence Scale for Children (3rd edition) (WISC-III) ${ }^{17}$ was used for evaluation of IQ. We identified 88 individuals with a full-scale IQ of 70 or over, all of whom were singletons and clinically diagnosed as having autistic-spectrum disorder, which was confirmed by scrutiny of case notes. Among the 88 initially identified, 4 individuals had a history of neurological disorders, mainly epilepsy, and were excluded from the analysis. The remaining 84 participants, with a mean full-scale scale IQ score of 100.0 (s.d.=16.8, range 71134), underwent a thorough clinical interview and were found to meet the criteria for at least one of the DSM-IV diagnoses under the category of pervasive developmental disorders. ${ }^{18}$ To ensure diagnostic precision, the Autism Diagnostic Interview Revised (ADI-R $)^{19}$ was also conducted to confirm the diagnosis in these 84 participants. Of these, 75 individuals were diagnosed with autistic disorder ( 13 females, 62 males), 5 with Asperger disorder ( 1 female, 4 males), and 4 with pervasive developmental disorder not otherwise specified (all males). In the present study, we regarded all 84 individuals as having autistic-spectrum disorder.

As a comparison group, we recruited unrelated healthy volunteers among university students and children of the hospital staff in order to efficiently assemble individuals without intellectual disability. Accordingly, 208 controls were included in the study who had no lifetime diagnosis of any psychiatric disorder, were singletons, and were confirmed to have had no clinically significant developmental delay.

All participants were Japanese and born in Japan. Written informed consent was obtained from each participant, and also from the parent or guardian in cases in which the participant was a minor (under 18 years old). This study protocol was approved by the Hamamatsu University Hospital ethics committee.

\section{Measures}

From computerised data derived from the Mother and Child Health Handbook, we obtained information regarding parents' ages at the time of the birth of the participants, birth order, and householder's occupation as an indicator of socio-economic status. Birth order was divided into first- and later-born. Socioeconomic status was classified into 'high' and 'middle and low' based on the occupational status of the household. ${ }^{20}$ Parental ages were first treated as continuous variables, and then were divided into tertiles according to their distributions. In the sample studied, no individuals had missing information on parental ages, birth order, socio-economic status or age and gender.

The age of the mother and father may be related to autistic traits running in families, which has been referred to as a 'broader phenotype of autism' in a previous study. ${ }^{21}$ Here, we interviewed both or either of the parents using a short version of a family history interview method ${ }^{22}$ in order to determine whether parents and siblings of the participants had such a trait, and defined a 'broader phenotype' of autistic-spectrum disorder as having any two of the deficits among three domains of characteristics of autistic-spectrum disorder (i.e. communication, social behaviours and stereotyped behaviours).

\section{Analyses}

For the statistical analyses, we used STATA, version 9.2. Twosample Wilcoxon rank sum test and chi-squared test were adopted for the descriptive analyses. Unadjusted odds ratios (ORs) and 95\% confidence intervals (CIs) for the risk of autistic-spectrum disorder in relation to parental age were first estimated using logistic regression analyses, and, then, in the regression models, age, gender, birth order, socio-economic status, and the other parent's age were adjusted for as potential confounding factors. However, preliminary analysis revealed no significant difference in the distribution of socio-economic status between the two groups $(P=0.26)$, indicating that socio-economic status can be omitted to increase precision. ${ }^{23}$ Thus, we computed adjusted ORs with adjustment for age, gender, birth order, and the other parent's age in the final model of the logistic regression analysis.

\section{Results}

\section{Characteristics of participants}

As shown in Table 1, the median age significantly differed between individuals with high-functioning autistic-spectrum disorder and controls.

Significantly more males were included among individuals with autistic-spectrum disorder than among controls. There was a significant difference in the distribution of birth order between groups; the proportion of first-born was higher in the group with autistic-spectrum disorder.

The median value of paternal age at birth was significantly higher in those with autustic-spectrum disorders than controls (Table 1). We then restricted the age range of the control group to 5-27 years $(n=193)$, which was identical to that for the autistic-spectrum disorder group, and we compared paternal age between the groups. With this restriction, the difference between the two groups remained the same: the median age was 31 years for the group with autistic-spectrum disorder and 30 years for the control group; $P=0.048$ (not shown in the table).

\section{Parental age treated as continuous}

In the logistic regression analysis, in which no covariates were included, a significant 1.8-fold increased risk was evident for every 10 -year advance in paternal age at birth $(\mathrm{OR}=1.80, P=0.048$; upper part of Table 2). When only gender was adjusted for, the estimate of OR did not change substantially ( $\mathrm{OR}=1.93, P=0.04$, not shown in the table). Then, adjustment for age, gender and birth order slightly attenuated the association $(\mathrm{OR}=1.73$, $P=0.15$ ). Finally, adjustment of age, gender, birth order and maternal age at birth provided a 2.5 -fold increased risk associated with every 10-year advance in paternal age, which fell short of statistical significance $(P=0.06)$. We confirmed that inclusion of socio-economic status in this final model did not change the results.

As regards maternal age at birth, there was no significant difference between the two groups $(P=0.71)$. Nor was there increased risk of autistic-spectrum disorder associated with every 10 -year advance in maternal age at birth $(\mathrm{OR}=1.06, P=0.87)$. Non-significant ORs of high-functioning autistic-spectrum disorder associated with advanced maternal age at birth remained unchanged after controlling for confounding factors (upper part of Table 2).

\section{Parental age treated as categorical}

This analysis was conducted by categorising parental age into three levels (Table 2, lower half). In the unadjusted model, an increased risk of autistic-spectrum disorder was observed in the intermediate paternal age class $(29-32$ years, $O R=1.30)$ and in the highest paternal age class ( 33 years or over, $\mathrm{OR}=1.75$ ) relative to the reference paternal age class (less than 29 years). Likelihood ratio statistics (LRS) for the linear trend showed that one-level increase in age class corresponded to a 1.3 -fold increased risk, 


\begin{tabular}{|c|c|c|c|}
\hline & $\begin{array}{l}\text { Autistic-spectrum disorder } \\
\qquad n=84\end{array}$ & $\begin{array}{c}\text { Controls } \\
n=208\end{array}$ & Statistics \\
\hline \multicolumn{4}{|l|}{ Age, years } \\
\hline Mean (s.d.) & $16.2(5.9)$ & $21.7(5.5)$ & \\
\hline Median (range) & $15(5-27)$ & $22(5-34)$ & $Z=6.84, P<0.001^{\mathrm{a}}$ \\
\hline Male, $n(\%)$ & $70(83)$ & $104(50)$ & $\chi^{2}=27.6$, d.f. $=1, P<0.001$ \\
\hline First-born, $n(\%)$ & $61(73)$ & $98(47)$ & $\chi^{2}=15.7$, d.f. $=1, P<0.001$ \\
\hline High socio-economic status, $n$ (\%) & $18(21)$ & $33(16)$ & $\chi^{2}=1.28$, d.f. $=1, P=0.26$ \\
\hline \multicolumn{4}{|l|}{ Paternal age at offspring's birth, years } \\
\hline Mean (s.d.) & $31.9(4.1)$ & $30.8(4.3)$ & \\
\hline Median (range) & $31(24-45)$ & $30(22-52)$ & $Z=2.14, P=0.046^{a}$ \\
\hline \multicolumn{4}{|c|}{ Maternal age at offspring's birth, years } \\
\hline Mean (s.d.) & $28.3(3.4)$ & $28.3(3.8)$ & \\
\hline Median (range) & $27(21-36)$ & $27(19-39)$ & $Z=0.38, P=0.71^{\mathrm{a}}$ \\
\hline
\end{tabular}

Table 2 Odds ratios (ORS) and 95\% confidence intervals (Cls) for the association between parental age at offspring's birth and the risk of high-functioning autistic-spectrum disorder in offspring

\begin{tabular}{|c|c|c|c|}
\hline & $\begin{array}{l}\text { Unadjusted } \\
\text { OR }(95 \% \mathrm{Cl})\end{array}$ & $\begin{array}{l}\text { Adjusted for age, gender } \\
\text { and parity, OR }(95 \% \mathrm{Cl})\end{array}$ & $\begin{array}{l}\text { Adjusted for age, gender, parity } \\
\text { and other parent's age, OR }(95 \% \mathrm{Cl})\end{array}$ \\
\hline \multicolumn{4}{|c|}{ Parental age as continuous variable ${ }^{a}$} \\
\hline Paternal age & $1.80(1.00-3.21)$ & $1.73(0.82-3.63)$ & $2.54(0.96-6.72)$ \\
\hline Maternal age & $1.06(0.54-2.10)$ & $0.98(0.40-2.41)$ & $0.48(0.15-1.56)$ \\
\hline \multicolumn{4}{|c|}{ Parental age (years) as categorical variable } \\
\hline Paternal 33+ & $1.75(0.92-3.34)$ & $1.87(0.82-4.24)$ & $3.09(1.17-8.16)$ \\
\hline Paternal 29-32 & $1.30(0.69-2.45)$ & $1.73(0.82-3.66)$ & $2.28(1.02-5.11)$ \\
\hline Paternal $<29^{b}$ & 1.0 & 1.0 & 1.0 \\
\hline Maternal 31+ & $0.90(0.48-1.71)$ & $0.69(0.29-1.63)$ & $0.38(0.13-1.06)$ \\
\hline Maternal 28-30 & $0.88(0.47-1.67)$ & $0.76(0.34-1.70)$ & $0.52(0.21-1.25)$ \\
\hline Maternal $<28^{b}$ & 1.0 & 1.0 & 1.0 \\
\hline
\end{tabular}

although this trend failed to reach statistical significance (LRS for linear trend $=2.91$, d.f. $=1, P=0.09)$.

However, the full model, in which all confounders were allowed for, revealed a significant linear increase in risk across paternal age classes. A one-level advance in paternal age class corresponded to a 1.8 -fold increase in risk $(\mathrm{OR}=1.78,95 \% \mathrm{CI}$ $1.10-2.88$, LRS for the linear trend $=5.62$, d.f. $=1, P=0.02$ ).

On the other hand, no significant linear trend was observed in relation to maternal age, even when confounding factors were controlled for

The narrow age-band width for the intermediate class of the paternal age group is arguable. We therefore categorised paternal age into four classes: $<25,25-29,30-34$, and 35 years or over. This analysis revealed a linear trend across the paternal age groups: unadjusted $\mathrm{OR}=1.41 \quad(95 \%$ CI $1.02-1.95, P=0.04)$ and fully adjusted $\mathrm{OR}=1.85$ (95\% CI $1.13-3.03, P=0.02$, adjusted for age, gender, parity and maternal age), which lends support to the linearity of the paternal age effect. It should be noted, however, that the confidence interval of the estimate of OR for the age group of 35 years or over in comparison with the reference youngest age group inevitably became wide $(\mathrm{OR}=14.3,95 \% \mathrm{CI}$ $1.19-173.3)$.

Lack of IQ data among the control group is also of concern in that mean full-scale IQ may be higher in the control group, even though individuals in the autistic-spectrum dirsorder group were shown to be high functioning. We additionally analysed the data by restricting it to those participants with autistic-spectrum disorder who had a full scale $I Q \geqslant 100$ (i.e. those with very high-functioning autistic-spectrum disorder, $n=34$ ). We found that a linear trend across three paternal age classes was far more conspicuous in those with very high-functioning autisticspectrum disorder than in the whole sample of participants with high-functioning autistic-spectrum disorder; for the former, one-level advance in age classes corresponds to a 2.8 -fold increase in risk $(\mathrm{OR}=2.81,95 \% \mathrm{CI} 0.97-8.12)$, whereas for the latter it corresponded to a 1.8 -fold increase.

\section{Paternal age in relation to a 'broader phenotype' of autistic-spectrum disorder}

Among 84 individuals with high-functioning autistic-spectrum disorder, 10 had a first-degree relative with a 'broader phenotype' of autistic-spectrum disorder; 1 individual had an elder brother with the phenotype, and the remaining 9 participants had fathers with such a phenotype. Paternal age at birth was compared between the 9 individuals whose fathers had an autistic phenotype and 74 individuals with autistic-spectrum disorder without the phenotype. There was no significant difference in paternal age at birth in the two subgroups: the median parental age was 31 years for familial participants and 31 years for non-familial participants (two-sample Wilcoxon rank sum test: $z=0.20, P=0.84)$. 


\section{Discussion}

\section{Comparisons with other studies}

In our Japanese sample, we found an advanced paternal age at birth to be associated with an increased risk of high-functioning autistic-spectrum disorder. This finding is in accord with that of previous studies that have been conducted exclusively in Western countries. ${ }^{1-3}$ The strength of the association, as assessed by a change in the odds ratio, was increased with adjustment for age, gender, birth order and maternal age at birth, indicating that these factors were indeed confounding factors for the association between paternal age at birth and the risk of high-functioning autistic-spectrum disorder. We did not find any association between maternal age at birth and the development of autisticspectrum disorder in our study. This negative finding of an maternal age effect in our study is in line with two recent studies, ${ }^{1,2}$ which have demonstrated the paternal age effect, but not the maternal age effect.

This is the first study to explore the paternal age effect on the risk of autistic-spectrum disorder, by focusing on individuals with autistic-spectrum disorder without cognitive impairment (i.e. high-functioning autistic-spectrum disorder). As noted above, advanced paternal age at birth has been reported to be linked with relatively low IQ and the risk of cognitive impairment in offspring. ${ }^{13,14}$ Because previous studies exploring parental age effects did not exclude those with autistic-spectrum disorder with cognitive impairment, ${ }^{1-3,5,7,24}$ the association found in those studies could be ascribed to the inclusion of such individuals. In this regard, our study demonstrated a direct relationship between advanced paternal age and the risk of high-functioning autisticspectrum disorder in offspring.

\section{Cultural factors influencing the findings}

Fathering age, together with age at marriage, may to some extent be dependent on a socio-cultural context. In particular, delayed age at marriage has become increasingly common in recent decades in high-income countries. In this regard, the participants with autistic-spectrum disorder in our study were relatively younger than the controls, suggesting that more recently born individuals were included in the autistic-spectrum disorder group or that older individuals were oversampled in the control group. However, we then matched the age range of the controls to that of the participants with autistic-spectrum disorder and the significantly higher paternal age for these participants remained the same. This finding, in addition to the findings from Western countries, points to the involvement of non-cultural factors in the phenomenon.

\section{Behavioural factors of fathers influencing the findings}

Age at marriage and subsequent fathering age may also be affected by individual behavioural patterns. Of concern is the behavioural consequence of vulnerable fathers with an autistic trait; they may be less sociable and may marry later than those without such a trait, possibly resulting in paternal age at birth higher in the autistic probands with familial loading of autistic-spectrum disorder than in those without it. When the fathering age was compared between individuals whose father had an autisticspectrum disorder phenotype and those whose father did not have such a phenotype, no significant difference was observed. This result provides evidence against an explanation that the paternal age effect could be attributable solely to heritable behavioural traits in the fathers of individuals with autistic-spectrum disorder.

\section{Negative results of maternal age effect}

We failed to replicate the association observed between advanced maternal age and the risk of autistic-spectrum disorder, as reported by two population-based studies. ${ }^{7,25}$ Of note is that these studies did not control for paternal age at birth when maternal age effect was analysed. Naturally, paternal and maternal age at birth are strongly correlated with each other. In this regard, it is worth noting that more recent studies, including ours, all showed that the maternal age effect vanished or was diminished after adjustment for paternal age at birth. ${ }^{2,3,5}$ Therefore, the maternal age effect shown by the two earlier studies may be an erroneous reflection of an association between paternal age at birth and the risk of autistic-spectrum disorder. However, because this is the first report in non-Western countries, it remains possible that the maternal age effect may be demonstrable in some, but not all, populations and may be related to societal and cultural factors.

\section{Other explanations for the findings}

Pregnancy and birth complications have been suggested to be associated with autistic-spectrum disorder. ${ }^{24}$ If advanced paternal age were related to such complications, which in turn are associated with autistic-spectrum disorder, then the parental age effect would be explained by the complications. In our study we conducted an analysis in which history of pregnancy and birth complications was controlled for using data from the Mother and Child Health Handbook. Addition of pregnancy and birth complications to the logistic regression model did not substantially change the results for the association found (OR for one-level advance in paternal age class $=1.80$ ), but confidence intervals of the estimates of the association became wider, indicating that the sample size employed is not large enough to reliably allow for pregnancy and birth complications.

Paternal age effect has been discussed in the literature in connection with de novo mutation; ${ }^{2,3}$ new mutations can occur in the paternal germ line as the father gets older. This idea is derived from findings suggesting that autosomal dominant diseases are linked with advanced paternal age ${ }^{26}$ new mutations in sperm are passed on to the offspring of fathers of advanced age. Although the contribution of new mutations to the occurrence of autistic-spectrum disorder remains unknown, a recent study has demonstrated that copy number variation is increased in individuals with autism, which in turn suggests that de novo germ line mutation is a risk factor of autistic-spectrum disorder in sporadic cases. ${ }^{27}$

Since advanced paternal age is not a rare event and has become ever more common in recent years, the actual percentage of risk of autistic-spectrum disorder in the general population attributable to exposure to advanced paternal age may be high. Further studies elucidating the mechanism underlying the association between advanced paternal age at birth and the risk of autistic-spectrum disorder are warranted.

\section{Limitations and strengths of the study}

First, the participants with high-functioning autistic-spectrum disorder in our sample were clearly not representative of the entire population of people with autistic-spectrum disorder. Small sample size is a concern, resulting in limited statistical power.

Second, selection of controls needs to be considered; the control group did not undergo any screening for autistic-spectrum 
disorder and IQ testing, and we may have overlooked some individuals in the control group with undetected autisticspectrum disorder. Basically, this misclassification would bias the results towards the null hypothesis. However, there remains a concern over possibly lower IQ in the group with autisticspectrum disorder. Although the additional analyses using only participants with autistic-spectrum disorder of full-scale IQ $\geqslant 100$ found that the paternal age effect was even more pronounced in these individuals with very high-functioning autistic-spectrum disorder, this result did not compensate for the lack of information on IQ in the control group. With a more suitably matched control group, firmer conclusions could be drawn.

Third, since it is noted that our sample of individuals with autistic-spectrum disorder was made up of more first-born individuals, one may raise a concern that parents who have a trait of autistic-spectrum disorder would have fewer children than those who do not, which would in turn account for more firstborn individuals in the case group and would be related to an advanced paternal age as an artefact. Unfortunately, we have no data on sibling size. Thus, to address this question, we have performed an additional analysis that was restricted to individuals who were not the first born (23 cases and 110 controls). This approach is not optimum, but any effect arising from 'more first-born' in the autistic-spectrum disorder group would be minimised. This result showed that one-level advance in paternal age class corresponded to a 1.70 -fold increase in risk $(\mathrm{OR}=1.70)$, which is almost identical to that obtained from the original analysis $(\mathrm{OR}=1.78)$. Thus, this suggests that the strength of association did not differ substantially after eliminating first-born individuals. Based upon this result, the issue of more first-born individuals in the autistic-spectrum disorder group compared with the control group does not appear to have had a major impact on the findings. However, due to the lack of information of sibling size and issue on the autistic-spectrum disorder phenotype assessment as discussed below, further studies are needed.

Lastly, although there was no difference in paternal age at birth in fathers of individuals with autistic-spectrum disorder with and without a broader autism phenotype, the proportion of fathers with the broad autism phenotype among fathers of autisticspectrum disorder probands was smaller (11\%) than would otherwise be expected (as high as $26 \%$ ). ${ }^{28}$ Since not all fathers of the participants were directly interviewed in this study, subtle traits may have been overlooked among the participants' fathers who were themselves labelled as not having the autistic-spectrum disorder phenotype. The question remains to be answered in a more appropriate manner as to whether paternal age at birth is related to behavioural traits in fathers that are genetically linked with autistic-spectrum disorder.

The diagnostic procedures used in the present study yielded unique insights in this field. We collected clinically validated diagnostic information via a rigorous assessment process, combined with the use of the ADI-R. Other studies exploring parental age in individuls with autistic-spectrum disorder have relied upon a registered diagnosis without the use of such interviews. Another strength is the inclusion of age, gender, birth order, and maternal age in the analyses, providing a significant improvement in the final model. Therefore, our findings indicate that birth order is indeed an important confounding factor when the relationship between paternal age at birth and the risk of autistic-spectrum disorder is examined.

In summary, the results of a Japanese sample supported the association between advanced paternal age at birth and an increased risk of high-functioning autistic-spectrum disorder in offspring.
Kenji J. Tsuchiya, MD, PhD, Osaka Hamamatsu Joint Research Center for Child Mental Development, and Department of Psychiatry and Neurology, Hamamatsu University School of Medicine, Hamamatsu, Japan; Kaori Matsumoto, MA Taishi Miyachi, MD, PhD, Osaka Hamamatsu Joint Research Center for Child Mental Development, Hamamatsu University School of Medicine, Hamamatsu, Japan; Masatsugu Tsujii, PhD, Osaka Hamamatsu Joint Research Center for Child Mental Development, Hamamatsu University School of Medicine, Hamamatsu, and Faculty of Sociology, Chukyo University, Nagoya, Japan; Kazuhiko Nakamura, MD, PhD, Shu Takagai, MD, PhD, Masayoshi Kawai, MD, PhD, Atsuko Yagi, MD, PhD, Kimie Iwaki, MD, Shiro Suda, MD, PhD, Department of Psychiatry and Neurology, Hamamatsu University School of Medicine, Hamamatsu, Japan; Genichi Sugihara, MD, PhD, Osaka Hamamatsu Joint Research Center for Child Mental Developme, MD, PhD, Osaka Hamamatsu Jont Research Center for Child Mental Development, MD, PhD, Department of Psychiatry and Neurology, Hamamatsu University School of Medicine, Hamamatsu, Japan; Hideo Matsuzaki, MD, PhD, Osaka Hamamatsu Joint Research Center for Child Mental Development, Hamamatsu University School of Medicine, Hamamatsu, Japan; Yoshimoto Sekine, MD, PhD, Katsuaki Suzuki, MD, PhD, Department of Psychiatry and Neurology, Hamamatsu University School of Medicine, Hamamatsu, Japan; Toshirou Sugiyama, MD, PhD, Aichi Children's Health and Medical Center, Obu, Japan; Norio Mori, MD, PhD, Department of Psychiatry and Neurology, Hamamatsu University School of Medicine, Hamamatsu, Japan; Nori Takei, MD, PhD, MSc, Osaka Hamamatsu Joint Research Center for Child Mental Development and Department of Psychiatry and Neurology, Hamamatsu University School of Medicine, Hamamatsu, Japan, and Division of Psychological Medicine, Institute of Psychiatry, London, UK.

Correspondence: Nori Takei, Osaka Hamamatsu Joint Research Center for Child Mental Development (OHJRC-CMD), Hamamatsu University School of Medicine, Handayama 1 Higashiku, Hamamatsu 431-3192, Japan. Email: ntakei@hama-med.ac.jp

First received 4 Sep 2007, final revision 2 May 2008, accepted 21 May 2008

\section{Acknowledgements}

This study was financially supported by a Grant-in-Aid for Scientific Research from the Ministry of Education, Culture, Sports, science and Technology, Japan. K.J.T. is a recipient of a Grant-in-Aid for Science Research (C) (2) (No. 18591280). N.T. is a recipient of a Grantin-Aid for Science Research (B) (2) (No. 14370288), and a grant for the National Center for Child Health and Development (19KOU-3) from the Ministry of Health, Labour and Welfare, Japan.

\section{References}

1 Lauritsen MB, Pedersen CB, Mortensen PB. Effects of familial risk factors and place of birth on the risk of autism: a nationwide register-based study. $J$ Child Psychol Psychiatry 2005; 46: 963-71.

2 Reichenberg A, Gross R, Weiser M, Bresnahan M, Silverman J, Harlap S, Rabinowitz J, Shulman C, Malaspina D, Lubin G, Knobler HY, Davidson M, Susser E. Advancing paternal age and autism. Arch Gen Psychiatry 2006; 63: 1026-32.

3 Croen LA, Najjar DV, Fireman B, Grether JK. Maternal and paternal age and risk of autism spectrum disorders. Arch Pediatr Adolesc Med 2007; 161: 334-40.

4 Kolevzon A, Gross R, Reichenberg A. Prenatal and perinatal risk factors for autism: a review and integration of findings. Arch Pediatr Adolesc Med 2007; 161: 326-33.

5 Larsson $\mathrm{HJ}$, Eaton WW, Madsen KM, Vestergaard M, Olesen AV, Agerbo E, Schendel D, Thorsen P, Mortensen PB. Risk factors for autism: perinatal factors, parental psychiatric history, and socioeconomic status. Am J Epidemiol 2005; 161: 916-25.

6 Lord C, Mulloy C, Wendelboe M, Schopler E. Pre- and perinatal factors in high-functioning females and males with autism. J Autism Dev Disord 1991; 21: 197-209.

7 Glasson EJ, Bower C, Petterson B, de Klerk N, Chaney G, Hallmayer JF. Perinatal factors and the development of autism: a population study. Arch Gen Psychiatry 2004; 61: 618-27.

8 Bhasin TK, Schendel D. Sociodemographic risk factors for autism in a US metropolitan area. J Autism Dev Disord 2007; 37: 667-77.

9 Mannerkoski MK, Aberg LE, Autti TH, Hoikkala M, Sarna S, Heiskala HJ. Newborns at risk for special education placement: a population-based study. Eur J Paediatr Neurol 2007; 11: 223-31.

10 Vestergaard M, Mork A, Madsen KM, Olsen J. Paternal age and epilepsy in the offspring. Eur J Epidemiol 2005; 20: 1003-5.

11 Sipos A, Rasmussen F, Harrison G, Tynelius P, Lewis G, Leon DA, Gunnell D. Paternal age and schizophrenia: a population based cohort study. BMJ 2004; 329: 1070.

12 Tsuchiya KJ, Takagai S, Kawai M, Matsumoto H, Nakamura K, Minabe $Y$, Mori N, Takei N. Advanced paternal age associated with an elevated risk for 
schizophrenia in offspring in a Japanese population. Schizophr Res 2005; 76: 337-42.

13 Auroux MR, Mayaux MJ, Guihard-Moscato ML, Fromantin M, Barthe J, Schwartz D. Paternal age and mental functions of progeny in man. Hum Reprod 1989; 4: 794-7

14 Malaspina D, Reichenberg A, Weiser M, Fennig S, Davidson M, Harlap S, Wolitzky R, Rabinowitz J, Susser E, Knobler HY. Paternal age and intelligence: implications for age-related genomic changes in male germ cells. Psychiatr Genet 2005; 15: 117-25.

15 Prevalence of autism spectrum disorders-autism and developmental disabilities monitoring network, six sites, United States, 2000. MMWR Surveill Summ 2007; 56: 1-11.

16 Wechsler D. Wechsler Adult Intelligence Scale - Revised. The Psychological Corporation, 1981.

17 Wechsler D, Golombok S, Rust J. Wechsler Intelligence Scale for Children Third Edition UK (WISC-IIIUK). The Psychological Corporation, 1992.

18 American Psychiatric Association. Diagnostic and Statistical Manual of Mental Disorders. APA, 1994.

19 Lord C, Rutter M, Le Couteur A. Autism Diagnostic Interview-Revised: a revised version of a diagnostic interview for caregivers of individuals with possible pervasive developmental disorders. J Autism Dev Disord 1994; 24 659-85.

20 Hollingshead AB, Redlich FC. Social Class and Mental Illness: A Community Study. John Wiley \& Sons, 1958.
21 Bolton $\mathrm{P}$, Macdonald H, Pickles A, Rios P, Goode S, Crowson M, Bailey A, Rutter M. A case-control family history study of autism. J Child Psychol Psychiatry 1994; 35: 877-900.

22 Piven J, Palmer $P$, Jacobi D, Childress D, Arndt S. Broader autism phenotype: evidence from a family history study of multiple-incidence autism families. Am J Psychiatry 1997; 154: 185-90.

23 Hosmer D, Lemeshow S. Applied Logistic Regression. John Wiley \& Sons, 1989.

24 Hultman CM, Sparen $\mathrm{P}$, Cnattingius S. Perinatal risk factors for infantile autism. Epidemiology 2002; 13: 417-23.

25 Eaton WW, Mortensen PB, Thomsen PH, Frydenberg M. Obstetric complications and risk for severe psychopathology in childhood. J Autism Dev Disord 2001; 31: 279-85.

26 Risch N, Reich EW, Wishnick MM, McCarthy JG. Spontaneous mutation and parental age in humans. Am J Hum Genet 1987; 41: 218-48.

27 Sebat J, Lakshmi B, Malhotra D, Troge J, Lese-Martin C, Walsh T, Yamrom B, Yoon S, Krasnitz A, Kendall J, Leotta A, Pai D, Zhang R, Lee YH, Hicks J, Spence SJ, Lee AT, Puura K, Lehtimaki T, Ledbetter D, Gregersen PK, Bregman J, Sutcliffe JS, Jobanputra V, Chung W, Warburton D, King MC, Skuse D, Geschwind DH, Gilliam TC, Ye K, Wigler M. Strong association of de novo copy number mutations with autism. Science 2007; 316: 445-9.

28 Hurley R, Losh M, Parlier M, Reznick JS, Piven J. The broad autism phenotype questionnaire. J Autism Dev Disord 2007; 37: 1679-90.

\section{Autism}

\section{Simon Baron-Cohen}

Autism Spectrum Conditions (ASC) occur in 1\% of the population, are strongly heritable, and result from atypical neurodevelopment. Classic autism and Asperger Syndrome (AS) share difficulties in social functioning, communication and coping with change, alongside unusually narrow interests. IQ is average or above in AS with average or even precocious age of language onset. Many areas within the 'social brain' are atypical in ASC. ASC has a profile of impaired empathy alongside strong 'systemising'. Hence, ASC involves disability (when empathy is required) and talent (when strong systemising would be advantageous). Psychological interventions that target empathy by harnessing systemising may help. 